\section{Symptomatic giant cavernous hemangioma of the liver in a 42-year-old man}

\author{
Abdu Hamisu Dambatta \\ Department of Radiology, Faculty of \\ Clinical Sciences, College of Health \\ Sciences, Bayero UniversIty, Kano, \\ Nigeria
}

\begin{abstract}
Most cases of liver hemangioma are asymptomatic and discovered incidentally on liver ultrasound or computed tomography scan. Giant cavernous hemangioma $(\mathrm{GCH})$ are however clinically distinct from smaller asymptomatic ones and may be confused with primary or metastatic malignancy. Symptomatic GCH of the liver are rare. The aim of the study was to show the rare case of discovered incidentally on liver ultrasound or computed tomography scan. GCH are however clinically distinct from smaller asymptomatic ones and may be confused with primary or metastatic malignancy. Symptomatic giant cavernous hemangiomas $(\mathrm{GCH})$ of the liver are rare. ${ }^{3}$ Hence, a case is presented in a 43-year-old man using ultrasound and contrast enhanced computerized tomography (CT) scan.
\end{abstract} symptomatic giant cavernous hemangioma of the liver which may be confuse with primary or metastatic malignancy. The patient's images (abdominal ultrasound and computed tomography scans) and case file were reviewed and summarized. The subject matter of giant cavernous hemangioma was reviewed in the literature. The index case was discussed and compared with literature. We report a 42-year-old man who presented with a 2-year history of right upper abdominal pain and 6-month history of chest pain. No history of jaundice, body swelling or previous history of blood transfusion. No history of smoking or alcohol consumption. The patient is a known diabetic. Abdominal ultrasound scan showed hepatomegaly with a huge well defined oval shaped mixed echogenic mass lesion with lobulated margins occupying $4^{\text {th }}$ and $5^{\text {th }}$ segments of liver, measuring $84.9 \times 111 \mathrm{~mm}$ in size suggestive of adenoma. The remaining hepatic parenchyma was normal. No intrahepatic biliary dilatation was seen. Initial histological examination revealed adenoma. Repeat histology done later however revealed hepatitis. Abdominal CT scan was later carried out and showed hepatomegaly but with no discernible mass lesion on precontrast images. Contrast enhanced images however, showed a fairly rounded mass lesion with peripheral enhancement and delayed filling-in at the venous phase, seen in the superior aspect of the right lobe extending to the dome. The intrahepatic vasculature and biliary ducts are not dilated. A diagnosis of cavernous hemangioma was made based on criteria of delayed (centripetal) filling-in. The patient however was lost to follow up. Hepatic hemangioma can be giant and symptomatic and despite its rarity, may still be encountered in practice.

\section{Introduction}

Liver hemangioma is the most common benign tumor of the liver. ${ }^{1}$ It is composed of cavities lined with endothelial cells and filled with blood. Giant cavernous hemangioma $(\mathrm{GCH})$ is defined as a lesion greater than $4 \mathrm{~cm}$ in diameter. ${ }^{1}$ Various reports indicate an incidence of cavernous hemangioma in the general population of up to $7.3 \%$. It is more common in women than in men and can be seen in any age group. ${ }^{2}$ Most cases of liver hemangioma are asymptomatic and

\section{Case Report}

S.A is a 42-year-old man who presented to the Medical Specialty Clinic of Aminu Kano Teaching Hospital (AKTH), Kano with a 2-year history of right upper abdominal pain and 6-month history of chest pain. No history of jaundice, body swelling or previous history of blood transfusion. No history of smoking or alcohol consumption. The patient is a known diabetic. On examination, the patient was afebrile, anicteric, not pale, not dehydrated. Abdominal and other systemic review were essentially normal.

Abdominal ultrasound scan showed hepatomegaly with a huge well defined oval shaped mixed echogenic mass lesion with lobulated margins occupying $4^{\text {th }}$ and $5^{\text {th }}$ segments (Figure 1), suggestive of adenoma. It measured $84.9 \times 111 \mathrm{~mm}$ in size. The remaining hepatic parenchyma was normal. No intrahepatic biliary dilatation was seen. Other organs were normal.

Laboratory investigations revealed normal liver function test, alpha feto protein and urea and electrolyte. Hepatitis B and C surface antigens were non-reactive. Initial histological examination revealed adenoma. Repeat histology done later however revealed hepatitis.

Abdominal CT scan was later carried out and showed hepatomegaly but with no discernible mass lesion on precontrast images (Figure 2). Contrast enhanced images however, showed a fairly rounded mass lesion with peripheral enhancement (Figure 3) and delayed filling-in at the venous phase (Figure 4), seen in the superi-
Correspondence: Abdu Hamisu Dambatta, Department of Radiology, Faculty of Clinical Sciences, College of Health Sciences, Bayero UniversIty, Kano, Nigeria

E-mail: najibdambatta@yahoo.com

Key words: Symptomatic, Giant, Cavernous hemangioma.

Conflict of interest: the author declares no potential conflict of interest.

Funding: none.

Received for publication: 9 August 2018.

Revision received: 5 January 2019.

Accepted for publication: 30 May 2019.

This work is licensed under a Creative Commons Attribution NonCommercial 4.0 License (CC BY-NC 4.0).

(C) Copyright: the Author(s), 2019

Licensee PAGEPress, Italy

Pyramid Journal of Medicine 2019; 2:18

doi:10.4081/pjm.2019.18

or aspect of the right lobe extending to the dome. The intrahepatic vasculature and biliary ducts are not dilated. A diagnosis of cavernous hemangioma was made. The patient was however lost to follow up.

\section{Discussion}

Cavernous hemangioma is the most common primary liver tumor; its occurrence in the general population ranges from $0.4-20 \%{ }^{4}$ Cavernous hemangiomas arise from the endothelial cells that line the blood vessels and consist of multiple, large vascular channels lined by a single layer of endothelial cells and supported by collagenous walls. Originating from the mesodermal layer, these lesions represent congenital, non-neoplastic hamartomatous proliferation of vascular endothelial cells. ${ }^{5}$ The tumor derives blood supply from the hepatic artery. ${ }^{6}$

Usually, they occur as solitary lesions, similar to this case. However, it may be multiple in $50 \%$ of patients. ${ }^{4}$ No lobar predilection exists. Similar hemangiomatous lesions may occur in other organs, ${ }^{5}$ but in this case it was confined to the liver only as all the other organs were spared. Hemangiomas typically measure less than 5 $\mathrm{cm}$; those larger than $4-5 \mathrm{~cm}$ are called Giant hemangiomas. In the present case it measured $8.5 \times 11.1 \mathrm{~mm}$ in size thus qualifying it as a giant hemangioma.

Approximately $80 \%$ of hemangiomata are of the cavernous type. ${ }^{5}$ Giant Hepatic 
cavernous hemangiomas are rare benign hepatic tumors, and occur with a strong female prevalence. ${ }^{3,7,8}$ Even though this case is the cavernous type, it occurred in a male in contradistinction to the norm. Unlike the less common capillary type, which are generally smaller in size, more frequently multiple and do not generally cause symptoms, cavernous hemangioma can grow to reach large sizes and may become symptomatic as in this case, with symptoms including abdominal pain or nausea which may arise through mass effect. Clinical features of polymyalgia rheumatica have also been described in association with a giant cavernous hemangioma, but these were not seen in this case. These features also resolved following resection of the lesion. ${ }^{5}$

Hemorrhage and thrombosis are occasional complications. Similarly, co-existence of platelet sequestration and Giant Hepatic cavernous hemangiomas is seen in Kassabach-Merritt syndrome. ${ }^{3}$ The lesion may be associated with focal nodular hyperplasia.

In most cases, radiological studies are sufficient for a definitive diagnosis of typical cavernous hemangiomas without the need for pathological examination. With increasing application and resolution of abdominal imaging modalities, hemangiomata are detected more frequently. On ultrasound, the most common appearance of hemangioma is that of a well-delineated hyperechoic but sometimes can be hypoechoic ${ }^{9}$ mass with faint acoustic enhancement. ${ }^{3}$ On computed tomography, the pattern of centripetal nodular enhancement with progressive filling as well as isoattenuation with the blood vessels on unenhanced and contrast-enhanced images is peculiar to hemangioma ${ }^{3}$ as was seen in this case. The Fluid-fluid level may be seen in cavernous hemangioma due to the separation of blood cells and serous fluid as there is an extremely slow flow of blood in cavernous hemangioma of the liver.

MR imaging has better sensitivity and specificity for diagnosing hemangioma. ${ }^{3}$ On T2 weighted imaging, hemangiomas have higher signal intensity which may be modified by the presence of a hypointense central scar. ${ }^{10}$ On $\mathrm{T} 1$ weighted imaging, enhancement after gadolinium administration is seen. However due to non-availability of MRI facility at AKTH, it could not be performed on this patient.

Similarly, nuclear Medicine has an important role in confirmation of diagnosis of liver hemangioma. ${ }^{99 \mathrm{~m} T c-l a b e l e d ~ R B C}$ scan is not only a highly sensitive and specific investigation but is also simple, noninvasive and cost effective for diagnosing cavernous hemangioma. Early phase imaging reveals a focal photopenic defect, which fills in centripetally with delayed imaging over a 30 to 50 minutes time interval. ${ }^{3}$ Like MRI, it is not available at AKTH.

At angiography, the feeding vessels of the hemangioma are of normal caliber, except those in the large tumors. ${ }^{4}$ During the late arterial/hepatic parenchymal phases, a dense, nodular pattern of opacification of the dilated vascular spaces persists into the venous phase. Angiography however was not performed as the US and CT scans were fairly sufficient to diagnose the hepatic hemangioma.

Liver function tests (LFT) are generally normal in the presence of giant haemangioma, ${ }^{\mathbf{5}}$ although LFT abnormalities have been reported as a consequence of biliary compression by the mass. In the present

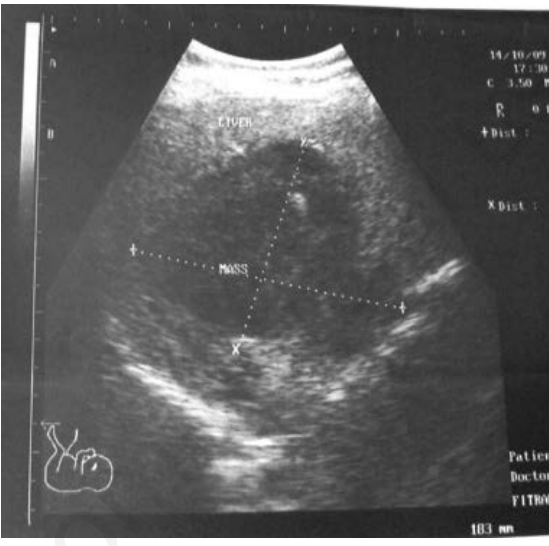

Figure 1. Abdominal sonogram of the liver showing an inhomogenously hypoechoic mass lesion that has focal eccentric calcification within it with lobulated margins occupying the $4^{\text {th }}$ and $5^{\text {th }}$ segments.

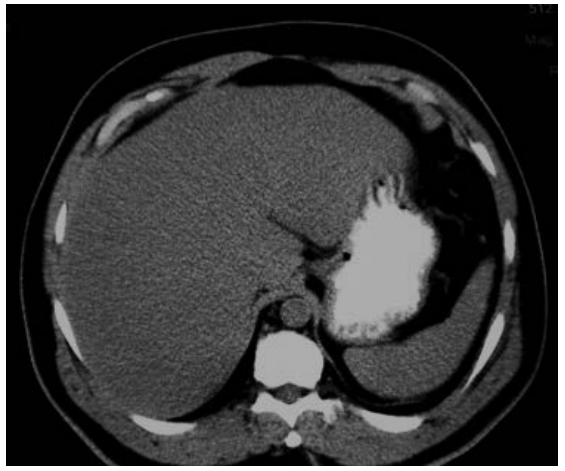

Figure 2. Axial non-contrast enhanced computed tomography scan of the abdomen showing hepatomegaly. No discernible mass lesion is however seen.

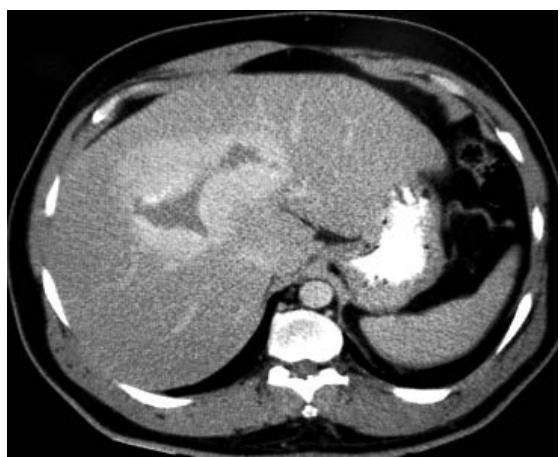

case, the LFT was normal despite the size of the mass.

Complications of hepatic hemangioma include: Kasabach-Merritt syndrome, intrahepatic bleed, and Budd-Chiari syndrome. ${ }^{5}$ Traumatic rupture is also a recognized but rare complication with a handful of cases described in the literature. KasabachMerritt syndrome is characterized by thrombocytopenia and consumptive coagulopathy in association with large hemangiomata, and may prompt intervention. Platelet trapping in the hemangioma is thought to result in activation of platelets and the clotting cascade, resulting in a consumptive coagulopathy.

The successful management of giant hemangioma depends on: (1) confirming the diagnosis; (2) determining whether the lesion requires surgical treatment; (3) deter-

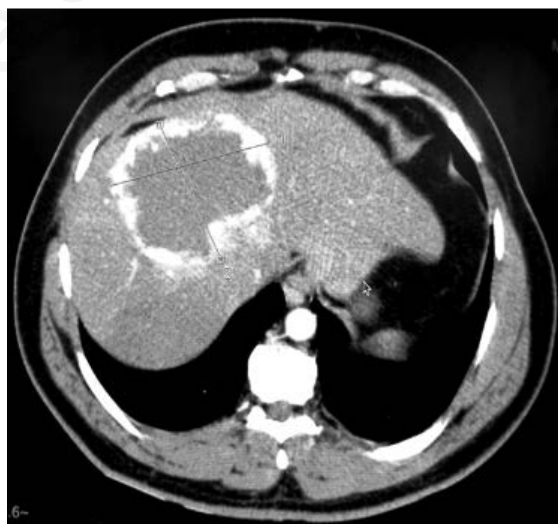

Figure 3. Post contrast axial computed tomography scan of the abdomen showing fairly rounded peripherally enhancing hepatic mass lesion in the arterial phase.

Figure 4. Post contrast axial computed tomography scan of the abdomen in the late venous phase showing centripetal fillin (arrows) of contrast medium. 
mining the optimal type of surgery, and (4) avoiding unnecessary surgical intervention.

In the face of a large body of evidence indicating a benign and uncomplicated natural history for the majority of hemangiomas, including giant hemangioma, a policy of non-operative management will be the optimal approach for the majority of patients. In the minority of cases that present as a surgical emergency due to hemorrhage, rupture, thrombosis and infarction, surgical management may be the only appropriate course of action. ${ }^{\mathbf{5}}$ There is also a role for the elective surgical management of giant hemangioma, albeit in a highly selected group of patients. Having taken all possible steps to ensure that symptoms are attributable to the hemangioma, surgical resection may be justified on grounds of intractable symptoms. Despite apparently satisfactory surgical management, symptoms persist in approximately $25 \%$ of patients following resection of seemingly symptomatic haemangioma. ${ }^{5}$

\section{Conclusions}

A diagnosis of a giant hepatic hemangioma was made using ultrasound and computed tomography scan in a 42-year-old man.

\section{References}

1. Saadeh S, Mullen KD, Carey W, et al. Infarcted Giant Liver Hemangioma Presenting with Signs of Acute Inflammation. Pract Gastroenterol 2003:68-72.

2. Bree RL, Schwab RE, Glazer GM, Bennett DF. The varied appearances of hepatic cavernous hemangiomas with sonography, computed tomography, magnetic resonance imaging and scintigraphy. RadioGraphics 1987;7: 1153-75.

3. Amin UM, Mahmood R, Shafique M. Peripheral puddling of contrast-classical enhancement pattern of giant cavernous hepatic hemangioma revealed on computed tomography in an adult male.
Pakistan Armed Forces Med J 2008;2.

4. Prasad SR. Cavernous Liver Hemangioma Imaging. Available from: http://emedicine.medscape.com/article/364860-overview\#showall.

5. Duxbury MS, Garden OJ. Giant Haemangioma of the Liver: Observation or Resection? Dig Surg 2010;27:7-11.

6. Cha EY, Kim KW, Choi YJ, et al. Multicystic cavernous haemangioma of the liver: Ultrasonography, CT, MR appearances and pathological correlation. Br J Radiol 2008;81:37-9.

7. Sahani DV, Kalva SP. Imaging the Liver. Oncologist 2004;9:385-97.

8. Brogna A, Ferrara R, Bucceri AM, et al. An atypical case of hepatic Cavernous haemangioma. Cases J 2009;2:181.

9. Bell DJ, Weerakkody Y. Hepatic haemangioma. Radiopedia. Available from:

https://radiopaedia.org/articles/hepatic-haemangioma-3

10. Klotz T, Montoriol PF, Da Ines D, et al. Hepatic haemangioma: Common and uncommon imaging features. Diagn Intervent Imag 2013;94:849-59. 